\title{
Análisis de la motivación del estudiante de educación superior participante en una propuesta de evaluación formativa
}

\section{Analysis of the motivation of higher education students participating in a formative assessment experience}

\author{
María del Carmen Campos-Mesa ${ }^{1 *}$, Gloria González-Campos ${ }^{1}$ y Carolina Castañeda-Vázquez ${ }^{1}$
}

${ }^{I}$ Facultad de Ciencias de la Educación, Universidad de Sevilla (España).

\begin{abstract}
Resumen: El objetivo del presente trabajo consiste en analizar la influencia de una innovación educativa basada en la evaluación formativa, sobre la motivación del alumnado universitario hacia una asignatura del Grado de Educación Primaria. Para ello, 91 estudiantes de la Universidad de Sevilla participaron en un proyecto de innovación docente durante el curso 20182019, donde se aplicó un proceso general de evaluación formativa, así como otros recursos didácticos y tecnológicos, como el uso de la gamificación y mandos interactivos de respuesta, entre otros. Al finalizar la intervención el alumnado respondió el Cuestionario de Estrategias de Aprendizaje y Motivación (CEAM II) para analizar, entre otras variables, la motivación del alumnado. Los datos obtenidos dejan entrever altos niveles de motivación, tanto intrínseca como extrínseca, lo que supone una valoración positiva para considerar esta innovación como una buena práctica educativa y seguir trabajando en esta línea.

Palabras clave: Educación Física, evaluación formativa, estudiantes universitarios, tecnología educativa, innovación educativa.
\end{abstract}

Abstract: The main aim of this study is to analyze the influence of an educational innovation based on formative assessment, on the motivation of university students towards a subject of the Degree in Primary Education. To this end, 91 students from University of Seville participated in a teaching innovation project during the 2018-2019 academic year, where a general process of formative assessment as well as other didactic and technological resources were applied, such as use of gamification and interactive response commands, among others. At the end of the intervention students answered the Spanish version of Motivated Strategies for Learning Questionnaire (CEAM II) in order to analyze, among other variables, students' motivation. The findings show high levels of motivation, both intrinsic and extrinsic. Therefore, the data obtained suggest this experience can be considered as a good educational practice and they encourage us to continue working in this line.

Keywords: Physical Education, Formative Assessment, University Students, Educational Technology, Educational Innovation.

\section{Introducción}

Este estudio surge del trabajo continuado por y para la excelencia de la Educación Superior. Tras varios estudios sobre evaluación continua virtual (Campos-Mesa, 2015; CamposMesa, 2016), la evaluación continua en el aula para favorecer la motivación e implicación del alumnado utilizando estrategias de gamificación (González-Campos, Castañeda-Vázquez y Campos-Mesa, 2018), nos sigue motivando como docentes para saber cómo proceder en el proceso de enseñanza-aprendizaje en la Educación Superior. Siendo conscientes de que la evaluación es uno de los elementos curriculares que desde el punto de vista del alumnado afecta a otros elementos del currículum (Álvarez, 2003; Biggs, 2005; Bonsón y Benito, 2005; Dochy et al., 2002; López, 2009; Sanmartí, 2007; Santos Guerra, 2003), planteamos el siguiente estudio sobre la evaluación formativa en Educación Superior.

Partiendo del contexto educativo que tendrán los egresados graduados de Educación Primaria, según la legislación educativa andaluza y española de ámbito estatal en vigor en Andalucía, la Orden de 4 de noviembre de 2015, por la que

Dirección para correspondencia [Correspondence address]: María del Carmen Campos Mesa. E-mail: mccampos@us.es se establece la ordenación de la evaluación del proceso de aprendizaje del alumnado de Educación Primaria en la Comunidad Autónoma de Andalucía, se hace referencia a que la evaluación, como parte esencial del proceso de enseñanzaaprendizaje, no puede limitarse a la mera comprobación de que los conocimientos han sido memorizados, haciendo alusión a que el proceso de la evaluación debe realizarse mediante instrumentos y procedimientos que promuevan, de manera paulatina, la autogestión del esfuerzo personal y el autocontrol del alumnado sobre el propio proceso de aprendizaje.

Estamos convencidos de que el egresado reproduce en muchos casos el proceso de enseñanza que ha recibido a lo largo de su Formación Inicial del Profesorado. En definitiva, al finalizar su formación, serán los profesionales los que aplicarán las leyes de educación vigente. Por lo tanto, López-Pastor y Pérez-Pueyo (2017: 52) se plantean:

“¿Cómo es posible que el profesorado en formación inicial desarrolle buenas competencias en evaluación si nunca utiliza y experimenta procesos, técnicas e instrumentos de evaluación formativa durante dicha formación inicial; o si nunca ha tenido que enfrentarse a instrumentos y procesos de autoevaluación, evaluación entre iguales o evaluación compartida; 
o nunca ha tenido que elaborar instrumentos de evaluación específicos para situaciones concretas de aprendizaje?"

Actualmente como apunta el Real Decreto 126/2014, de 28 de febrero, por el que se constituye el currículo básico de la Educación Primaria, se establece que la comprobación del grado de adquisición de las competencias y el logro de los objetivos de la etapa en las evaluaciones continuas y finales, así como que, los maestros evaluarán tanto los aprendizajes del alumnado como los procesos de enseñanza y su propia práctica docente.

Por todo lo expuesto, pensamos que la evaluación formativa, entendida como se concibe desde la Red de Evaluación Formativa y Compartida como "todo proceso de constatación, valoración y toma de decisiones cuya finalidad es optimizar el proceso de enseńanza-aprendizaje que tiene lugar, desde una perspectiva humanizadora y no como mero fin calificador" (Pérez et al., 2009: 35), es la más adecuada para llevar a cabo en los contextos de enseńanza aprendizaje, independientemente de nivel educativo.
Aunque se es consciente, como expresa Niscar (2012), de que por un lado la alta ratio del alumnado, entre otro orden de cosas, hace que se sobrecargue de trabajo al profesorado con este tipo de evaluación. Esto unido a que existen docentes que entienden que su papel consiste en la elaboración y aplicación de instrumentos con fines evaluativos (de medición) con base en los cuales establecer un juicio valorativo (Pérez et al., 2008), hace difícil la aplicación de este tipo de experiencias en Educación Superior. Realmente son las autoridades académicas las que deben tener en cuenta el incremento de trabajo que supone para el profesor algunas de estas innovaciones y reordenar la distribución de obligaciones docentes de los profesores.

Después de una revisión de la literatura sobre evaluación formativa, se entiende esta como un proceso. En este proceso no todos los autores coinciden en los pasos. En la tabla 1 se expone una comparativa entre los distintos pasos que describen diversos autores.

Tabla 1. Comparación de los pasos a seguir según autores en el proceso de evaluación formativa (Elaboración propia).

\begin{tabular}{|c|c|c|c|}
\hline $\begin{array}{l}\text { Pasos de Evaluación } \\
\text { Formativa }\end{array}$ & Smarter Balanced Assessment Consortium (2015) & Rosales (2014) & Pasek y Mejía (2017) \\
\hline 1 & $\begin{array}{l}\text { Aclarar el aprendizaje que se quiere lograr (implica establecer } \\
\text { metas de aprendizaje y criterios de éxito) }\end{array}$ & Identificar problemas & Exploración \\
\hline 2 & $\begin{array}{l}\text { Obtener evidencia de diversas fuentes en función de las } \\
\text { metas y criterios previos }\end{array}$ & Mostrar alternativas & Monitoreo \\
\hline 3 & $\begin{array}{l}\text { Interpretar la evidencia, es decir, dilucidar junto con los } \\
\text { estudiantes el significado de la información recabada para } \\
\text { determinar dónde ubican su aprendizaje respecto a las metas } \\
\text { y criterios previstos }\end{array}$ & $\begin{array}{l}\text { Detectar los obstácu- } \\
\text { los para superarlos }\end{array}$ & Resalta logros \\
\hline 4 & $\begin{array}{l}\text { Actuar sobre la base de la evidencia, o sea, realimentar el } \\
\text { proceso y decidir los pasos siguientes con el fin de avanzar } \\
\text { en el aprendizaje, tomando en cuenta dificultades, intereses } \\
\text { y preferencias de cada estudiante }\end{array}$ & & Controla \\
\hline 5 & & & Promueve la autoevaluación \\
\hline 6 & & & Promueve la coevaluación \\
\hline 7 & & & Ofrece realimentación \\
\hline
\end{tabular}

En su investigación, Pasek y Mejía (2017) tras analizar ocho docentes, encuentran que hay escasa aplicación de la evaluación formativa, justificando este hecho a que es poco sistemática. Por este motivo, configuraron un proceso general para realizar una evaluación formativa del aprendizaje consciente y metódico. Este proceso de evaluación formativa es el que llevamos a cabo en nuestro estudio, resumido en la figura 1. 


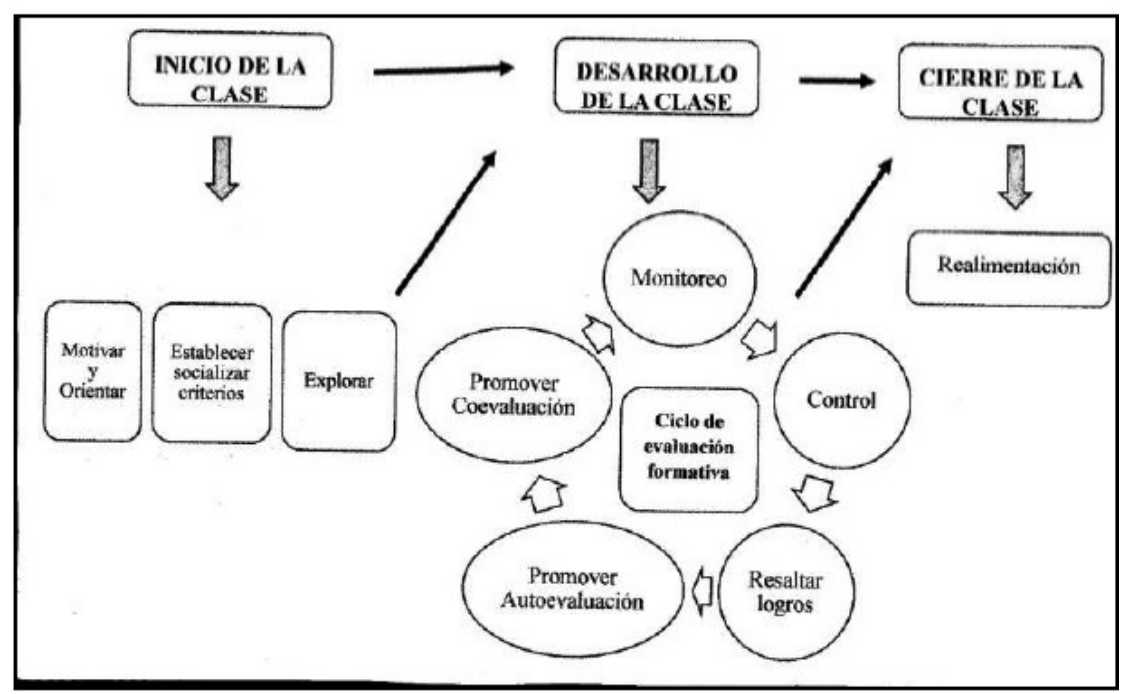

Figura 1. Esquema del proceso de evaluación formativa (Pasek y Mejía, 2017:186).

Por todo lo anterior, en la presente investigación se plantea como objetivo general, llevar a cabo el proceso de evaluación formativa descrito por Pasek y Mejía (2017) para conocer los niveles de motivación intrínseca y extrínseca del alumnado. Para ello, se proponen los siguientes objetivos específicos: elaborar y aplicar un proceso de evaluación formativa, y medir la motivación intrínseca y extrínseca del alumnado participante en este proceso.

\section{Material y método}

\section{Diseño y participantes}

Esta investigación se enmarca dentro de un diseño transversal y de corte descriptivo. La muestra de participantes está

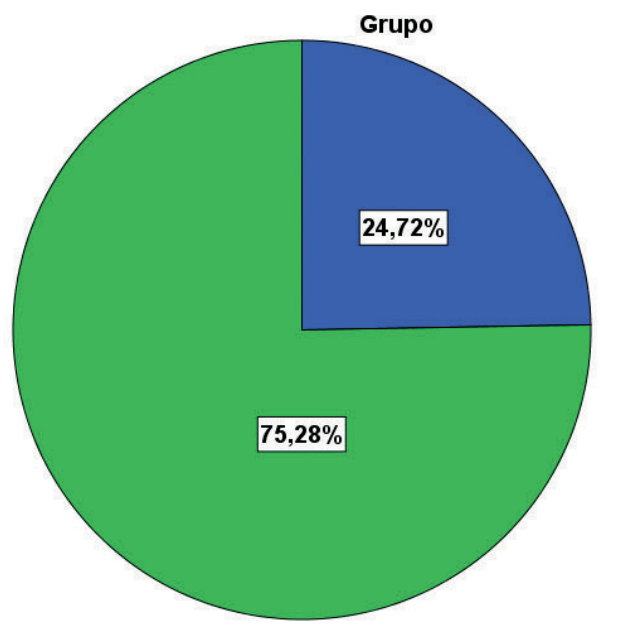

conformada por 91 estudiantes (15 hombres y 74 mujeres) de tercer año que cursan el Grado en Educación Primaria en la Facultad de Ciencias de la Educación de la Universidad de Sevilla, matriculados en el curso académico 2018/19 en la asignatura de "Fundamentos y currículum de Educación Física en Educación Primaria" del módulo obligatorio, en el turno de mańana, en los grupos 1 y 4 , seleccionados a través de muestreo por conveniencia. En la figura 2 se exponen las características de la muestra seleccionada.

Figura 2. Características de la muestra. 


\section{Procedimiento}

Desde el primer día de comienzo de la asignatura, el alumnado fue informado de la participación en la investigación y en qué consistiría. La asignatura se impartió por la profesora utilizando la evaluación formativa siguiendo los pasos que describen Pasék y Mejías (2017).

El primer día de clase se realizó una evaluación inicial con la herramienta educativa Educlick (mandos interactivos de respuestas), que formó parte del “paso 1 exploración”. Se impartieron los temas utilizando el material que el profesorado estimó conveniente (presentaciones PPT, vídeos, lecturas, debates, etc.), donde se llevó a cabo el "paso 2 monitoreo" por parte del profesorado de la asignatura. Al finalizar cada bloque de la asignatura, se desarrolló una tarea o actividad, llamada actijuego, esta formó parte del "paso 3 resalta logros", donde al alumnado se le premió con permanencia de nota en cada una de las actividades que realizaban. A continuación, se procedió al "paso 4 control", y gracias a las nuevas tecnologías, se utilizó una tablet en cada clase práctica para registrar y hacer un control de cada grupo y alumnado. En las clases prácticas se evaluó a través del "paso 5 instrumento de autoevaluación”. De cada tema, cada uno de los grupos tuvo que presentar al profesor un total de cinco preguntas tipo test con cuatro opciones de respuesta, esta actividad formó parte del "paso 6 coevaluación". Tras la finalización de todos los temas, se realizó una prueba con las preguntas que diseñó el alumnado y la corrigieron entre ellos. En todas las sesiones estuvo presente el "paso 7 retroalimentación".

En la tabla 2 se presentan los pasos y fases consideradas en la evaluación formativa llevada a cabo en esta investigación.

Tabla 2. Descripción de pasos a seguir en la evaluación formativa del estudio (Elaboración propia).

\begin{tabular}{lll}
\hline Pasos & Fases Descripción & \\
\hline 1 & Exploración & $\begin{array}{l}\text { Prueba con los mandos Educlick para registrar el conocimiento inicial del alumnado. Esta herra- } \\
\text { mienta fue subvencionada en la Convocatoria 2014-2015 para el Proyecto de Innovación Docente } \\
\text { titulado "Seguimiento en la web: una experiencia de evaluación continua virtual” por la Universi- } \\
\text { dad de Sevilla }\end{array}$ \\
\hline 2 & Monitoreo & En clase por parte del profesorado \\
\hline 3 & Resalta logros & $\begin{array}{l}\text { Utilización de estrategias de gamificación, llevadas a cabo en la convocatoria 2016-2017 en el Pro- } \\
\text { yecto de Innovación Docente titulado "Estrategias de gamificación, las TIC y evaluación continua } \\
\text { en el aula para favorecer la motivación e implicación del alumnado" }\end{array}$ \\
\hline 4 & Control & $\begin{array}{l}\text { Empleo de las nuevas tecnologías. Uso de tablets, para hacer los registros (clases prácticas en gim- } \\
\text { nasio) }\end{array}$ \\
\hline 5 & Promueve la autoevaluación & Instrumentos elaborados por el profesorado \\
\hline 6 & Promueve la coevaluación & $\begin{array}{l}\text { Instrumentos elaborados por los propios alumnos. Supervisado del profesor sobre la implicación del } \\
\text { alumnado en la asignatura }\end{array}$ \\
\hline 7 & Ofrece realimentación & Información por parte del profesor al alumnado en las clases \\
\hline
\end{tabular}

En la evaluación formativa también se tiene en cuenta la evaluación del docente y del contexto, para lo cual nos serviremos de diferentes instrumentos, como la opinión de los alumnos por medio de la asamblea y los cuestionarios de evaluación de la Actividad Docente del Profesorado de la Universidad de Sevilla que lleva a cabo el Vicerrectorado de Profesorado a través del Secretariado de formación y evaluación.

\section{Instrumento}

Para analizar la motivación del alumnado participante en el estudio se aplicó, tras la propuesta de evaluación formativa, el CEAM II (Roces, Tourón y González, 1995), traducción al castellano del MSLQ (Motivated Strategies for Learning Questionnaire), de Pintrich, Smith, García y Mckeachie (1991). Se trata de un autoinforme constituido por 81 ítems, que a su vez se divide en dos escalas: 31 ítems para la escala de motivación y 50 ítems para la escala de evaluación de estrategias de aprendizaje. A su vez, se diferencian seis subescalas motivacionales y siete subescalas de estrategias de aprendizaje. La escala de motivación está compuesta por: orientación a metas intrínsecas; orientación a metas extrínsecas; valor de la tarea; creencias de control y autoeficacia para el aprendizaje; autoeficacia para el rendimiento; y ansiedad. La escala de estrategias de aprendizaje está compuesta por: elaboración; aprovechamiento del tiempo y concentración; organización; ayuda; constancia; metacognición; y autointerrogación. Los alumnos responden de acuerdo a una escala likert de 7 puntos (1= no, nunca; ...; 7= sí, siempre). La fiabilidad del cuestionario es aceptable, pues las escalas de motivación y estrategias tienen un Alpha de Cronbach de 0.79 y 0.89 respectivamente (Nocito, 2013). 


\section{Análisis de datos}

El análisis de datos se efectuó mediante el programa SPSS versión 24.0 (IBM Corp, Armonk, NY, USA) para Windows. Se realizaron análisis descriptivos a través de frecuencias y porcentajes, así como se calculó la media (M) y la desviación típica (DT). Se analizó la normalidad de los datos a través de la prueba de Kolmogorov-Smirnov, obteniendo una puntuación de 0,00 y determinando así la distribución normal de la muestra. Para comparar las medias según el sexo y el grupo, se empleó la prueba $\mathrm{T}$ para muestras independientes.

\section{Resultados}

En la tabla 3 se muestran los datos de motivación del alumnado participante. Dichos datos dejan entrever un alto nivel de motivación alcanzado por el alumnado, tanto en las Metas Intrínsecas como Extrínsecas (pues en una escala de 7 puntos la media es cercana al 5 en ambos casos). Aunque apenas existen diferencias entre ambos tipos, se observa que la puntuación de la dimensión de Metas Intrínsecas es algo superior a las Metas Extrínsecas.

Tabla 3. Estadísticos descriptivos de las variables y dimensiones motivacionales.

\begin{tabular}{lccccc}
\hline Variables & Ítem No & M & DT & M (dimensión) & Alpha Cronbach \\
\hline \multirow{3}{*}{ Metas Intrínsecas } & 1 & 5,19 & 1,46 & & 0,68 \\
& 16 & 5,34 & 1,35 & 5,3 & \\
& 22 & 5,39 & 1,17 & & 0,67 \\
Metas Extrínsecas & 7 & 4,70 & 1,53 & & \\
& 11 & 5,26 & 1,40 & 4,8 & \\
\hline
\end{tabular}

El análisis extraído de la diferencia de medias según el grupo de pertenencia del alumnado se muestra en la tabla 4 .

Tabla 4. Diferencia de medias según el grupo de pertenencia del alumnado.

\begin{tabular}{lcccccc}
\hline \multirow{2}{*}{ Variables } & \multicolumn{2}{c}{ G1 } & G4 & & \\
\cline { 2 - 7 } & M & DT & M & DT & Levene & Sig. \\
\hline 1 & 5,36 & 1,43 & 5,16 & 1,49 & 0,65 & 0,58 \\
\hline 16 & 5,41 & 0,96 & 5,28 & 1,46 & 0,1 & 0,71 \\
\hline 22 & 5,29 & 0,96 & 5,44 & 1,24 & 0,08 & 0,6 \\
\hline Metas Intrínsecas & 5,33 & 0,72 & 5,3 & 1,14 & 0,04 & 0,6 \\
\hline 7 & 4,38 & 1,07 & 4,79 & 1,65 & 0,49 & 0,19 \\
\hline 11 & 5,27 & 1,12 & 5,24 & 1,49 & 1,53 & 0,92 \\
\hline 13 & 4,5 & 1,5 & 4,94 & 1,38 & 0,35 & 0,21 \\
\hline 30 & 3,95 & 1,53 & 4,53 & 1,79 & 0,31 & 0,19 \\
\hline Metas Extrínsecas & 4,52 & 0,54 & 4,86 & 1,18 & 0,01 & 0,08 \\
\hline
\end{tabular}

Según el análisis de los valores de la prueba de Levene, y teniendo en cuenta si se asume o no la igualdad de varianzas para tomar el valor de la significación según el caso, se observa en la tabla 4, que ninguna de las diferencias de medias calculadas, ya sea en las diferentes variables o dimensiones, llega a ser significativa, pues en ninguno de los casos el nivel de significación es menor a 0,05 $(p \leq 0,05)$.

El análisis de la diferencia de medias según el sexo del alumnado se describe en la tabla 5 .
Tabla 5. Diferencia de medias según el sexo del alumnado.

\begin{tabular}{lcccccc}
\hline \multirow{2}{*}{ Variables } & \multicolumn{2}{c}{ Hombre } & \multicolumn{2}{c}{ Mujer } \\
\cline { 2 - 7 } & $\mathrm{M}$ & $\mathrm{DT}$ & $\mathrm{M}$ & $\mathrm{DT}$ & Levene & Sig. \\
\hline 1 & 5,6 & 1,45 & 5,14 & 1,47 & 0,86 & 0,27 \\
\hline 16 & 5,53 & 1,5 & 5,27 & 1,33 & 0,41 & 0,5 \\
\hline 22 & 5,57 & 1,45 & 5,37 & 1,12 & 0,31 & 0,56 \\
\hline Metas Intrínsecas & 5,59 & 1,24 & 5,25 & 1,01 & 0,83 & 0,26 \\
\hline 7 & 5,13 & 1,6 & 4,6 & 1,52 & 0,9 & 0,22 \\
\hline 11 & 5,2 & 1,65 & 5,26 & 1,36 & 0,71 & 0,88 \\
\hline 13 & 4,93 & 1,58 & 4,81 & 1,39 & 0,4 & 0,75 \\
\hline 30 & 4,8 & 1,78 & 4,31 & 1,73 & 0,87 & 0,32 \\
\hline Metas Extrínsecas & 5,01 & 1,23 & 4,73 & 1,04 & 0,67 & 0,36 \\
\hline
\end{tabular}

Tras el estudio de los valores de la prueba de Levene, y teniendo en cuenta si se asume o no la igualdad de varianzas para tomar el valor de la significación según el caso, se observa en la tabla 5, que ninguna de las diferencias de medias calculadas, ya sea en las diferentes variables o dimensiones, llega a ser significativa, pues en ninguno de los casos el nivel de significación $p$ es menor a 0,05 .

\section{Discusión y conclusiones}

Durante la experiencia de la investigación se ha descubierto que la práctica difiere de la propuesta teórica, ya que muchas de las actividades que al final se plantearon fueron actividades de enseñanza-aprendizaje y no constituyendo pasos de 
un proceso, como sucedió con los "actijuegos", ya que no se realizaron las anotaciones pertinentes. En esta línea Pasék y Mejías (2017) manifestaban lo mismo, justificando en su estudio que se debe a la fase de monitoreo, ya que los profesores que participaron en la investigación no tomaron notas de la información que generaban y por tanto carecían de juicios de la actuación del estudiante.

Por otro lado, la autoevaluación y la coevaluación, pasos 5 y 6 respectivamente, fueron unos de los pasos más valorados por el alumnado en la puesta en común del último día de clase. El hecho de analizar el contenido para redactar preguntas, confirma que les obligó a entender y estudiar con detenimiento el mismo desde otro punto de vista. Esta idea coincide con las del estudio de Rodríguez et al. (2011), quienes concluyen que el uso de la autoevaluación en el contexto de las universidades favorece la participación activa de los es- tudiantes, y además supone un ejercicio de autorregulación y autorreflexión crítica sobre su grado de conocimiento y desarrollo de habilidades.

Respecto a la motivación, el proceso de evaluación formativa llevado a cabo, ha logrado altos niveles de motivación, tanto intrínseca como extrínseca, obteniéndose en ambos casos una media de 5 puntos en una escala del 1 al 7, aunque sería importante resaltar que las Metas Intrínsecas es algo superior a las Metas Extrínsecas, proporcionando mayor autorrealización y calidad en los procesos de enseñanzaaprendizaje. Se coincide con lo que plantean Llorens-Larto et al. (2016), como claves hacia un aumento de la motivación intrínseca describiendo las mismas como la autonomía, competencia y relaciones sociales, además de dar significado al trabajo que realizan, que en definitiva es lo que se ha conseguido en nuestro estudio.

\section{Referencias}

1. Álvarez-Méndez, J. M. (2003). La evaluación a examen. Ensayos críticos. Madrid: Miño y Dávila.

2. Biggs, J. (2005). Calidad del aprendizaje universitario. Madrid: Narcea

3. Bonsón, M. y Benito, Á. (2005). Evaluación y aprendizaje. En Á. Benito y A. Cruz (Eds.), Nuevas claves para la docencia universitaria en el espacio europeo de educación superior (pp. 87-100). Madrid: Narcea.

4. Campos-Mesa, M.C. (2015). Evaluación continua a través de Educlick. En III Congreso Internacional de Educación a través del Deporte. Jaén: Universidad de Jaén.

5. Campos-Mesa, M.C. (2016). Una experiencia de evaluación continua interactiva. En V Jornadas de Innovación Docente de la Facultad de Ciencias de la Educación "Investigación, experiencias y reflexión en docencia universitaria”. Sevilla: Universidad de Sevilla.

6. Dacal, G.G. (1976). Evaluación formativa. Aula abierta, (15), 30-35.

7. Dochy, F., Segers, M. y Dierick, S. (2002). Nuevas vías de aprendizaje y enseñanza y sus consecuencias: una era de evaluación. Revista de Docencia Universitaria, 2(2), 13-30.

8. González-Campos, G.; Castañeda-Vázquez, C. y Campos-Mesa, M.C. (2018). Continuous assessment and interactive response systems in higher education. Journal of Human Sport and Exercise, 13(3). Doi:10.14198/jhse.2018.133.17

9. Hamodi, C., López-Pastor, V.M. y López-Pastor, A.T. (2015). Medios, técnicas e instrumentos de evaluación formativa y compartida del aprendizaje en educación superior. Perfiles educativos, 37(147), 146-161.

10. López-Pastor, V.M. (coord.) (2009). Evaluación formativa y compartida en educación superior. Propuestas, técnicas, instrumentos y experiencias. Madrid: Narcea.

11. Llorens-Largo, F., Gallego-Durán, F. J., Villagrá-Arnedo, C. J., Compañ, P., Satorre-Cuerda, R. y Molina-Carmona, R. (2016). Gamificación del proceso de aprendizaje: lecciones aprendidas. Vaep-rita. 4(1), 25-32.

12. Orden de 4 de noviembre de 2015 , por la que se establece la ordenación de la evaluación del proceso de aprendizaje del alumnado de Educación Primaria en la Comunidad Autónoma de Andalucía. Boletín número 230. De 26 de noviembre de 2015 https://www.juntadeandalucia.es/
boja/2015/230/BOJA15-230-00011_00001414.pdf

13. Pasek de Pinto, E.P. y Mejía, M.T. (2017). Proceso general para la evaluación formativa del aprendizaje. Revista Iberoamericana de Evaluación Educativa, 10(1), 177-193.

14. Pérez, Á., Tabernero, B., López-Pastor, V.M., Ureńa, N., Ruiz, E., Capllonch, M., González, N. y Castejón, J. (2008). "Evaluación formativa y compartida en la docencia universitaria: la concreción de cuestiones/ clave para su aplicación en el camino hacia el Espacio Europeo de Educación Superior (EEES)". Revista de Educación, 347, 435-451.

15. Pérez, Á., Julián, J.A. y López-Pastor, V.M. (2009). Evaluación formativa y compartida en el Espacio Europeo de Educación Superior (EEES)", en Víctor Manuel López Pastor (coord.), Evaluación formativa y compartida en Educación Superior, Madrid, Narcea, pp. 19-43.

16. Real Decreto $126 / 2014$, por el que se constituye el currículo básico de la Educación Primaria. Boletín Oficial del Estado, 52. De 28 de febrero de 2013, pp. 19349 a 19420. https://www.boe.es/buscar/pdf/2014/BOEA-2014-2222-consolidado.pdf

17. Roces, C., Tourón, J. y González, M.C. (1995). Validación preliminar del CEAM II (Cuestionario de estrategias de aprendizaje y motivación II). Psicológica, 16 (3), 347-366.

18. Rodríguez, G., Ibarra, M.S. y Gómez, M.A. (2011). E-autoevaluación en la universidad: un reto para profesores y estudiantes. Revista de Educación, 356, 401-430. DOI: http://dx.doi.org/10.4438/1988-592Xre-2011-356-045.

19. Rosales, M. (2014). Proceso evaluativo: evaluación sumativa, evaluación formativa y Assesment su impacto en la educación actual. En Congreso Iberoamericano de Ciencia, Tecnología, Innovación y Educación (Vol. 4).

20. Sanmartí, N. (2007). 10 ideas clave. Evaluar para aprender. Barcelona: Grao.

21. Santos-Guerra, M. A. (2003). Una Flecha en la diana: la evaluación como Aprendizaje. Madrid: Narcea.

22. Smarter Balanced Assessment Consortium (2015). The formative assessment process. Recuperado de https://www.smarterbalanced.org 